\section{Utilizing Cyclopentadiene for the Design of New Polyenyne Motifs}

Gategory

Synthesis of

Materials and

Unnatural Products

\section{Key words}

conjugated polymers

enynes

Sonogashira coupling

Synthesis of poly(cyclopentadienylene ethynylene)s (PCEs)
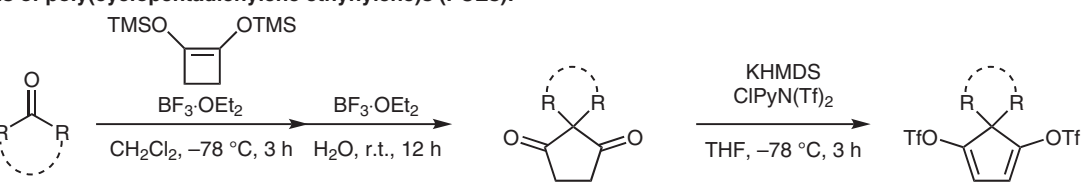

3MeCy-1

3-methylcyclohexanon

6-undecanone

Dipentyl-1 $53 \%$ yield

TMS $=3$ MeCy-2 $55 \%$ yield

Dipentyl-2 54\% yield

Cul

Cul

$60{ }^{\circ} \mathrm{C}, 12 \mathrm{~h}$

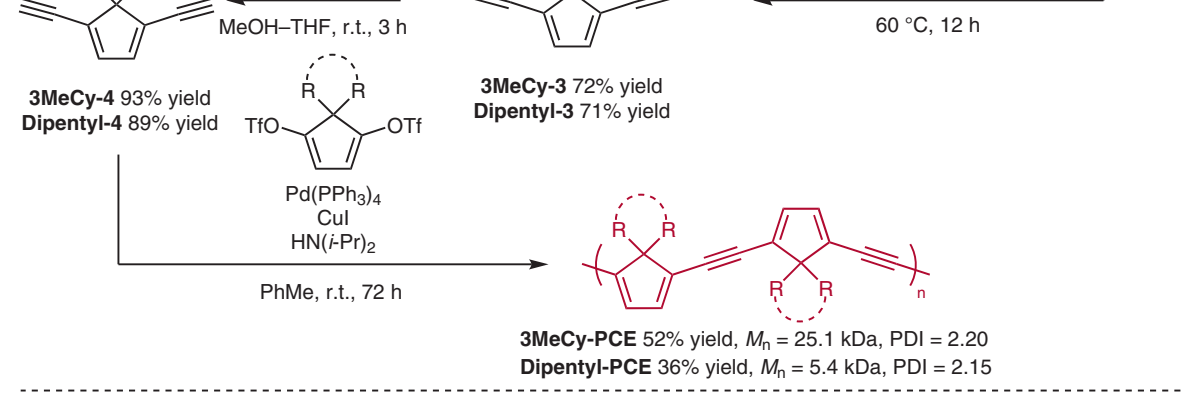

Synthesis of poly(p-phenylene ethynylene)/poly(cyclopentadienylene ethynylene) copolymers (PPECEs):

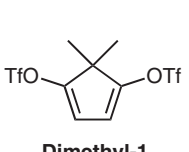

Dimethyl-1

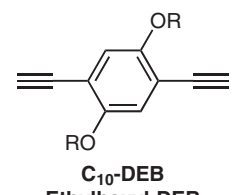

Ethylhexyl-DEB

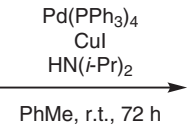

PhMe, r.t., $72 \mathrm{~h}$

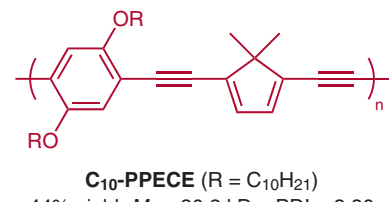

$44 \%$ yield, $M_{\mathrm{n}}=20.2 \mathrm{kDa}, \mathrm{PDI}=3.30$

Ethylhexyl-PPECE $(\mathrm{R}=2$-ethylhexyl) $54 \%$ yield, $M_{\mathrm{n}}=33.0 \mathrm{kDa}, \mathrm{PDI}=2.50$
Significance: Conjugated polyenynes are a unique class of polymers with backbones consisting entirely of $\mathrm{sp}$ - and nonaromatic $\mathrm{sp}^{2}$-hybridized carbon atoms. Despite its attractive electronic properties and potential application in materials science, the discovery of new polyenyne motifs remains challenging because of the dearth of suitable precursors and preparatory methods. Herein, Pietrangelo and co-workers report the synthesis of poly(cyclopentadienylene ethynylene)s (PCEs) and poly(p-phenylene ethynylene)/poly(cyclopentadienylene ethynylene) copolymers (PPECEs), in which a cyclopentadiene motif is incorporated into their monomeric structures.

SYNFACTS Contributors: Timothy M. Swager, Kosuke Yoshinaga Synfacts 2017, 13(09), 0923 Published online: 18.08.2017 DOI: 10.1055/s-0036-1591205; Reg-No.: S08417SF
Comment: Interestingly, the authors' results suggest that the solubilizing group structure of the PCEs has an effect on the polymer molecular weight and optical absorption profiles. In contrast, the solubilizing group structure of copolymer PPECEs has little effect on their optical absorption. Taken together, these results indicate that the cyclopentadiene constituents are responsible for the low-energy electronic transitions of PCEs. Evaluation of PPEs as semiconductors in organic electronic devices is under way. 